\title{
Phenolic profiling and quantitative determination of common sage (Salvia plebeia R. Br.) by UPLC-DAD-QTOF/MS
}

\author{
Seon-Hye Lee ${ }^{1}$ Heon-Woong Kim ${ }^{1} \cdot$ Min-Ki Lee ${ }^{1} \cdot$ Young Jin Kim $^{1} \cdot$ Gelila Asamenew ${ }^{1} \cdot$ Youn-Soo Cha ${ }^{2}$. \\ Jung-Bong $\mathrm{Kim}^{1}$ (D)
}

Received: 28 February 2018 / Revised: 4 April 2018 / Accepted: 7 April 2018 / Published online: 2 May 2018

(c) The Author(s) 2018

\begin{abstract}
Salvia plebeia $\mathrm{R}$. Br. is a medicinal herb that contains important active compounds such as flavonoid and phenolic acid which are responsible for remarkable biological properties. The phenolic composition of S. plebeia was quantitatively investigated using ultra-performance liquid chromatograph coupled with photodiode array detection and quadrupole time of flight mass spectrometry. A total of 16 flavonoids were classified into 10 flavones and 6 flavanones based on UV-spectra and ion fragmentation patterns. Individual flavonoids, including apigenin, luteolin, hispidulin, nepetin, and some flavanones, were glycosylated with glucose at the 5- or 7-position of flavonoid structure. The three major flavones were determined to be 6-hydroxyluteolin 7-O-glucoside [2,452.7 mg/100 g dry weight (DW)], hispidulin 7- $O$-glucoside $(2,281.0 \mathrm{mg} / 100 \mathrm{~g} \mathrm{DW})$ and nepetin $7-O$-glucoside $(2,002.6 \mathrm{mg} / 100 \mathrm{~g} \mathrm{DW})$. The six flavanones containing the hydroxyl and methoxy groups were determined and, among them, 5,7,3',4'- tetrahydroxy-6-methoxyflavanone 7-O-glucoside have the highest level $(938.3 \mathrm{mg} / 100 \mathrm{~g}$ DW). Two hydroxyl flavanone glycosides, 5,6,7,3', '4'- pentahydroxyflavanone 7-O-glucoside and 5,6,7,4'- tetrahydroxyflavanone 7-O-glucoside, were presumed to be newly identified compounds, on the basis of the library data from S. plebeia. In addition, higher concentration of rosmarinic acid was also identified.
\end{abstract}

\section{Graphical abstract}

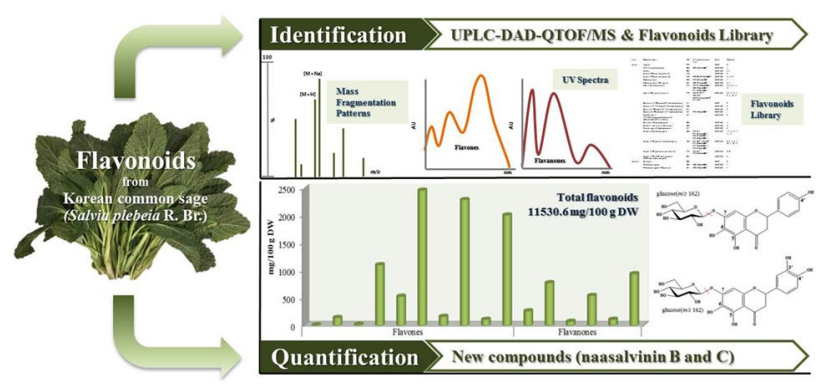

Keywords Salvia plebeia R. Br. · Flavonoids · Rosmarinic acid · UPLC-DAD-QTOF/MS

Electronic supplementary material The online version of this article (https://doi.org/10.1007/s00217-018-3076-6) contains supplementary material, which is available to authorized users.

Jung-Bong Kim

jungbkim@korea.kr

Extended author information available on the last page of the article

\section{Introduction}

Herbs are rich sources of flavonoids and are used as natural spices and traditional medicine ingredients [1]. Salvia is a medicinally important genus under the family Lamiaceae that contains interesting health-promoting phytochemicals, notably polyphenolic compounds. At present, $\sim 75$ types of simple phenolic acids have been isolated from 23 Salvia species, and 50 types of flavonoid have been isolated from 51 
Salvia species [2]. Common sage (Salvia plebeia $\mathrm{R}$. Br.) is a medicinally important species under the genus Salvia that is widely distributed throughout Korea, China, and India. This plant is used in Asian traditional medicines for the treatment of several illnesses because of its antimicrobial, diuretic, antiblastic, antipyretic, anti-inflammatory, and antidynous activities [3, 4]. S. plebeia has also been selected as a natural drug candidate among various Chinese traditional herbs owing to its strong antioxidant properties $[5,6]$.

S. plebeia is known in Korean folk medicine as 'BaemCha-Zu-Ki' and is used for the prevention and treatment of inflammatory diseases. This traditional claim is backed by scientific evidence from several animal model-based experiments. For example, the polyphenolic extract showed sedative and gastro-protective activities against two gastric lesion assay-based methods in mice [7]. Some researchers had reported the inhibitory effects of the aqueous extracts on immediate-type allergic reactions in rat. Moreover, a potent anti-inflammatory activity was observed from a methanol extract [8-10]. Phenolic acids, flavones, isoflavones, and their glycosides play vital roles in the anti-inflammatory and other wide-ranging pharmacological activities of S. plebeia $[3,9,11]$.

Flavonoids, known as a major group of phenolic compounds, affect the flavors and colors of plant-derived foods. They are categorized into flavanones, flavones, flavonols, isoflavones, and chalcones, and they possess protective and therapeutic effects against chronic diseases, such as cancer and coronary heart disease [12]. Many food resources, such as fruits, vegetables, and herbs, contain various flavonoids, such as quercetin, kaempferol, myricetin, luteolin, apigenin, naringenin, eriodictyol, and tangeretin [13]. Apigenin, luteolin, nepetin, hispidulin, and their glycosides are the major flavonoids found in the hydro-alcoholic extract of S. plebeia. The flavone classes are major phytochemicals responsible for the potent biological activity of the plant $[3,5,6,9,14-16]$. However, flavanone and other sub-classes of flavonoids are rarely reported, although they have significant pharmacological properties [17]. The previous studies revealed the potential biological activity of S. plebeia, and this effect is strongly related with the presence of polyphenolic compounds abundantly. Therefore, their component has been considered in developing nutraceutical ingredients as crude extracts and pure active compounds. The accurate quantification of the active compounds is a significant step for nutraceuticals and determining their physiological activities.

The aim of the present work was to characterize the phenolic compounds of $S$. plebeia using ultra-performance liquid chromatograph coupled with photodiode array detection and quadrupole time of flight mass spectrometry (UPLC-DAD-QTOF/MS). The flavonoids were quantified with an emphasis on the minor constituent flavanones. 5,6,7,4'-Tetrahydroxyflavanone 7-O-glucoside and $5,6,7,3^{\prime}, 4^{\prime}$-pentahydroxyflavanone 7 - $O$-glucoside are proposed as new compounds, among the identified flavanones.

\section{Materials and methods}

\section{Chemicals}

HPLC-grade solvents (acetonitrile, methanol, and water) were obtained from Fisher Scientific (Fair Lawn, NJ, USA). Formic acid was purchased from Junsei Chemical (Tokyo, Japan). Galangin (Sigma Co., St. Louis, MO, USA) and 2,4,5-trimethoxycinnamic acid (Extrasynthese, Genay, France) were used as the internal standard for flavonoids and phenolic acids, respectively.

\section{Plant material and extraction of phenolic compounds}

The aerial part of common sage (S. plebeia $\mathrm{R}$. Br.) was collected from Korea in 2015 and identified at the Paju Agricultural Technology Center, Korea. A voucher specimen (RDASPR14) was deposited in the Department of Agrofood Resources, National Institute of Agricultural Sciences, Rural Development Administration (Korea). The sample was freeze-dried in a deep freezer (PVTFD 10R, Ilsin Lab, Yangju, Korea), pulverized into powder and stored at $-60{ }^{\circ} \mathrm{C}$ prior to analysis.

The weighed powder $(1.0 \mathrm{~g})$ was suspended in $10 \mathrm{ml}$ of extract solvents (two types of solvent systems; methanol: water: formic acid, 50:45:5, v/v/v for flavonoids and methanol: water: formic acid, 80:15:5, v/v/v for phenolic acids) containing the internal standard and extracted for $5 \mathrm{~min}$ at $200 \mathrm{rpm}$ on an orbital shaker. The mixture was then centrifuged at $3000 \mathrm{rpm}$ at $4{ }^{\circ} \mathrm{C}$ for $10 \mathrm{~min}$. Each supernatant was immediately filtered through a syringe filter $(0.2 \mu \mathrm{m}$, PVDF; Whatman, Kent, England), and $0.5 \mathrm{ml}$ of phenolic extract was diluted by $4.5 \mathrm{ml}$ of water, resulting in $5 \mathrm{ml}$ of a phenolic-containing crude extract. The phenolic concentrate was then isolated from the crude extract using the solid phase extraction method with a Sep-pak $\mathrm{C}_{18}$ cartridge (Waters Co., Milford, MA, USA). The Sep-pak cartridges were activated by washing with $2 \mathrm{ml}$ of methanol, followed by washing with $2 \mathrm{ml}$ of water for conditioning. Then, the diluted flavonoid extract was loaded on a cartridge, and impurities were removed by washing with $2 \mathrm{ml}$ of water. Finally, total phenolic mixtures were eluted from the cartridge using $3 \mathrm{ml}$ of methanol. The phenolic eluent was concentrated using $\mathrm{N}_{2}$ gas, and then re-dissolved in $0.5 \mathrm{ml}$ of the extract solvents prior to analysis with UPLC-DAD-QTOF/MS. Internal standards were used follow as; $20 \mu \mathrm{g} / \mathrm{ml}$ of galangin and $250 \mu \mathrm{g} / \mathrm{ml}$ of 2,4,5-trimethoxycinnamic acid. All experimental analyses were performed in triplicate. 


\section{UPLC-DAD-QTOF/MS conditions}

The analysis of phenolic compounds was performed according to the Kim et al. [18] using UPLC-DAD-QTOF/MS system (Waters Co.) equipped with a Kinetex $1.7 \mu \mathrm{XB}^{-\mathrm{C}_{18}}$ $100 \AA$ column $(150 \times 2.1 \mathrm{~mm}$ i.d.; Phenomenex, Torrance, CA, USA) for flavonoids and a Cortecs UPLC T3 column $(150 \times 2.1 \mathrm{~mm}$ i.d.; Waters Co. $)$ for phenolic acids, respectively. The separation of flavonoids was carried out under the following instrumental conditions: flow rate of $0.3 \mathrm{ml} / \mathrm{min}$, the mobile phases $0.5 \%$ formic acid in water (solvent $\mathrm{A}$ ) and $0.5 \%$ formic acid in acetonitrile (solvent $\mathrm{B}$ ). The gradient condition used: 0 min 5\% (B), $20 \min 25 \%$ (B), $25 \min 50 \%$ (B), 30-32 min 90\% (B), 35-40 min 5\% (B). The chromatograms for flavonoids were acquired at 280 and $350 \mathrm{~nm}$ wavelengths for flavanones and flavones, respectively. Mass spectra were recorded in the range of $m / z, 200-1,200$ using electrospray ionization as the ionization source in positive ion mode. The MS settings used were: cone voltage, $30 \mathrm{~V}$; source temperature, $120{ }^{\circ} \mathrm{C}$; desolvation temperature $500{ }^{\circ} \mathrm{C}$; and desolvation $\mathrm{N}_{2}$ gas flow of $1,050 \mathrm{l} / \mathrm{h}$. Separation of phenolic acids was carried out under the following instrumental conditions: flow rate of $0.3 \mathrm{ml} / \mathrm{min}$, mobile phases $0.1 \%$ formic acid in water (solvent $\mathrm{A}$ ) and $0.1 \%$ formic acid in acetonitrile (solvent $\mathrm{B}$ ). The gradient condition used: 2-4 min 3\% (B), $15 \mathrm{~min} 4 \%$ (B), $20 \mathrm{~min} 6 \%$ (B), $30 \mathrm{~min}$ $11 \%$ (B), 40 min $15 \%$ (B), 42 min $25 \%$ (B), $47-48$ min $50 \%$ (B), 50-52 min $90 \%$ (B), 55-60 min 3\% (B). The chromatograms for phenolic acids were acquired at $280 \mathrm{~nm}$ and $320 \mathrm{~nm}$ wavelengths for hydroxybenzoic acid and hydroxycinnamic acid derivatives, respectively. Mass spectra were recorded in the range of $m / z 50-800$ using positive ion electrospray. The MS settings used were: cone voltage, $40 \mathrm{~V}$; source temperature, $120{ }^{\circ} \mathrm{C}$; desolvation temperature $500{ }^{\circ} \mathrm{C}$; and desolvation $\mathrm{N}_{2}$ gas flow of $1,020 \mathrm{l} / \mathrm{h}$. Instrument control, and data acquisition and evaluation for flavonoids and phenolic acids were performed using MassLynx V4.1 software. The identification of individual compounds from the sample was achieved by comparing UV-spectra and mass fragmentation patterns with the library database constructed from the literature (Table 1).

\section{Results and discussion}

\section{Identification and quantification of flavonoids from S. plebeia R. Br.}

The UPLC-DAD chromatogram revealed the presence of 10 peaks at $350 \mathrm{~nm}$ and 6 peaks at $280 \mathrm{~nm}$ (Fig. 1a, b). The chromatogram analysis, when compared with the constructed flavonoid library of S. plebeia showed possible novel compounds, glycosylation sites, and mass fragmentation patterns. The glycosylation of flavones and flavanones in S. plebeia occurred at the A-ring's 7-position, except for in luteolin 5-O-glucoside. Furthermore, the hydroxylated and methylated derivatives of apigenin and luteolin were found to be conjugated at the 6-positions of the flavonoid backbones. The four flavone aglycones were detected and assigned as luteolin (peak 12), nepetin (peak 13), apigenin (peak 15), and hispidulin (peak 16) based on previously reported MS data $[19,20]$. At retention times of 13.31 and 13.47 min, peaks 4 and 5 were produced and showed similar ion fragment patterns $(m / z, 471,449,287)$ by losing $\mathrm{m} / \mathrm{z} 162$, corresponding to a glucose or galactose moiety [21]. Conformation of peak 4 and 5 identification was performed by comparing the elution order of them with the literature. The 5-position glycosylation in the flavonoid ring system favor moderate polarity than that of the 7-position and result for faster retention time, hence, peak 4 and 5 were identified as luteolin 5-O-glucoside and luteolin 7-O-glucoside, respectively [22-24]. Peak 8 was assigned as apigenin 7-O-glucoside (cosmosiin) based on the parent ion $\mathrm{m} / \mathrm{z} 433$, sodium adducts ion $\mathrm{m} / \mathrm{z}, 455$, and the characteristic loss of the hexose fragment to obtain $\mathrm{m} / \mathrm{z} 271$ as the fragment ion [25]. Thus, apigenin 7-O-glucoside is reported for the first time from S. plebeia.

Peak 2 was a predominant peak observed on the UV spectrum, producing parent ion $\mathrm{m} / \mathrm{z}, 465$ and fragment ion $\mathrm{m} / \mathrm{z} 303$ after losing a glucose moiety (Fig. 2a). Study of hydroxyl flavones confirmed 6-hydroxyluteolin 7-O-glucoside existed in S. plebeia using nuclear magnetic resonance spectroscopy (NMR) [19]. The fragmentation pattern was similar to that of peak 2 , resulting in its identification as 6-hydroxyluteolin 7-O-glucoside.

A total of six flavanones were identified from the chromatogram of S. plebeia. In previous studies, the flavone class of flavonoids was the most abundant flavonoid compounds to be presented in S. plebeia; however, in the present study, unlike to the previous reports significant concentration of flavanones class were identified. A comparison of the molecular weights of peaks 6 and 9 showed $16 \mathrm{Da}$ increments that corresponded to peaks 1 and 3 (Table 2), respectively, owing to hydroxyl moiety substitutions at the position $3^{\prime}$ of the B-ring (Fig. 2c, d). Peaks 6 and 9 were assigned by comparing their spectroscopic data [14].

Peak 6 was identified as 5,7,3',4'-tetrahydroxy-6-methoxyflavanone $7-O$-glucoside, and peak 9 as $5,7,4^{\prime}$-trihydroxy-6-methoxyflavanone 7-O-glucoside. Compared with peaks $6(\mathrm{~m} / \mathrm{z} 481)$ and $9(\mathrm{~m} / \mathrm{z} 465)$, the parent ion of peaks $1(\mathrm{~m} / \mathrm{z}, 467)$ and $3(\mathrm{~m} / \mathrm{z}, 451)$ showed increases of $14 \mathrm{Da}$, respectively, suggesting an additional $\mathrm{CH}_{3}$ group instead of an $\mathrm{H}$ atom. Thus, these two compounds were presumed to be $5,6,7,3^{\prime}, 4^{\prime}$-pentahydroxyflavanone $7-O$-glucoside and $5,6,7,4^{\prime}$-tetrahydroxyflavanone 7 - $O$-glucoside, respectively. Their characteristic product ions, as shown in Table 1, 
Table 1 LC/MS and NMR library of S. plebeia $\mathrm{R}$. Br. from the literature

\begin{tabular}{|c|c|c|c|c|c|}
\hline Classes & Compound names & MW & UV Spectrum patterns $\left(\lambda_{\max }\right)$ & States & References \\
\hline \multirow[t]{21}{*}{ Flavones } & Apigenin & 270 & & NMR & {$[3]$} \\
\hline & $5,6,7,4^{\prime}$-tetrahydroxyflavone & 286 & $285,335 \rightarrow \mathrm{MeOH}[19]$ & NMR, MS & [19] \\
\hline & Luteolin & 286 & & NMR, MS & {$[11,16]$} \\
\hline & Luteolin 5-O-glucoside (galuteolin) & 448 & & NMR, MS & {$[14]$} \\
\hline & Luteolin 7- $O$-glucoside (cynaroside) & 448 & $239,269,340 \rightarrow \mathrm{MeOH}[22]$ & NMR, MS & {$[7,11,16,22]$} \\
\hline & 6-Hydroxyluteolin & 302 & $276,338 \rightarrow \mathrm{MeOH}[26]$ & NMR, MS & {$[21]$} \\
\hline & 6-Hydroxyluteolin 7-O-glucoside & 464 & $282,348 \rightarrow \mathrm{MeOH}[19]$ & NMR, MS & {$[7,19]$} \\
\hline & Nepetin (6-methoxyluteolin) & 316 & $\begin{array}{l}282,342 \rightarrow \mathrm{MeOH}[26] \\
271,348 \rightarrow \mathrm{MeOH}[19]\end{array}$ & NMR, MS & {$[11,14,16,19,26]$} \\
\hline & Nepetin 7-O-glucoside (nepitrin) & 478 & $\begin{array}{l}255,274,345[6] \\
284,340[26] \\
255,274,345 \rightarrow \mathrm{MeOH}[20] \\
271,348[19]\end{array}$ & NMR, MS & $\begin{array}{l}{[6,7,11,14,16,19} \\
\quad 20,26]\end{array}$ \\
\hline & Cirsimaritin (4',5-Dihydroxy-6,7-dimethoxyflavone) & 314 & & NMR & {$[3]$} \\
\hline & Jaceosidin (4',5,7-Trihydroxy-3',6-dimethoxyflavone) & 330 & & NMR, MS & {$[14]$} \\
\hline & Eupatilin (5,7-Dihydroxy-3',4',6-trimethoxyflavone) & 344 & & NMR, MS & [7] \\
\hline & Eupatorin (3',5-Dihydroxy-4',6,7-trimethoxyflavone) & 344 & $276,342 \rightarrow \mathrm{MeOH}[5]$ & NMR, MS & {$[5]$} \\
\hline & $\begin{array}{l}\text { Neocafhispidulin }\left(4{ }^{\prime}, 5,9,10 \text {-tetrahydroxy-6-methoxy- }\right. \\
\text { 12-methylchroman }[2,3-h] \text { flavone) }\end{array}$ & 434 & & NMR, MS & {$[14]$} \\
\hline & Scutellarein (6-hydroxyapigenin) & 286 & & NMR, MS & [19] \\
\hline & Sorbifolin (scutellarein 7-methyl ether) & 300 & & NMR, MS & {$[14]$} \\
\hline & Pectolinarigenin (6-methoxyacacetin) & 314 & & NMR, MS & [14] \\
\hline & Hispidulin (6-methoxyapigenin) & 300 & $\begin{array}{l}275,334[5] \\
274,334 \rightarrow \mathrm{MeOH}[26] \\
275,336 \rightarrow \mathrm{MeOH}[19]\end{array}$ & NMR, MS & $\begin{array}{l}{[5,11,14,16,19} \\
26]\end{array}$ \\
\hline & Hispidulin 7-O-glucoside (homoplantaginin) & 462 & $\begin{array}{l}274,330[6] ; 276,332[26] \\
274,330 \rightarrow \mathrm{MeOH}[20] \\
275,334 \rightarrow \mathrm{MeOH}[19]\end{array}$ & NMR, MS & $\begin{array}{l}{[6,11,14-16,19,} \\
20,26]\end{array}$ \\
\hline & Hispidulin 7-O-glucuronide (hispiduloside) & 476 & $\begin{array}{l}275,333[6] \\
275,333 \rightarrow \mathrm{MeOH}[20]\end{array}$ & NMR, MS & {$[6,20]$} \\
\hline & $\begin{array}{l}\text { Hispidulin 7-O-(6"'-O-acetyl)glucoside (6"-O-acetyl } \\
\text { homoplantaginin) }\end{array}$ & 504 & & NMR, MS & {$[14]$} \\
\hline \multirow[t]{6}{*}{ Flavanones } & Eriodictyol & 288 & & NMR & {$[3]$} \\
\hline & 6-Methoxynaringenin & 302 & $290,340 \rightarrow \mathrm{MeOH}[26]$ & NMR, MS & {$[26]$} \\
\hline & 6-Methoxynaringenin 7-O-glucoside & 464 & $282,342 \rightarrow \mathrm{MeOH}[26]$ & NMR, MS & {$[26]$} \\
\hline & $\begin{array}{l}\text { 5,7,4'-Trihydroxy-6-methoxyflavanone } 7-O \text {-glucoside } \\
\text { (naasalvinin A) }\end{array}$ & 464 & & NMR, MS & {$[14]$} \\
\hline & Filifolin $\left(5,7,3^{\prime}, 4^{\prime}\right.$-tetrahydroxy-6-methoxyflavanone) & 318 & & NMR & {$[3]$} \\
\hline & $\begin{array}{l}5,7,3^{\prime}, 4^{\prime} \text {-Tetrahydroxy-6-methoxyflavanone } 7-O \text {-gluco- } \\
\text { side (naasanone) }\end{array}$ & 480 & $285,343 \mathrm{sh} \rightarrow \mathrm{MeOH}[19]$ & NMR, MS & {$[14,19]$} \\
\hline \multirow[t]{2}{*}{ Flavonols } & Quercetin & 302 & & NMR & [7] \\
\hline & Isorhamnetin & 316 & & NMR & [3] \\
\hline Isoflavones & 2'-Hydroxy-5'-methoxybiochanin A & 330 & $\begin{array}{l}266,295 \rightarrow \mathrm{MeOH}[6,20] \\
253,259 \rightarrow \mathrm{MeOH}[6,20]\end{array}$ & NMR, MS & {$[6,20]$} \\
\hline
\end{tabular}

also demonstrated increases of $14 \mathrm{Da}$, suggesting that the methyl group attached to the phenolic hydroxyl group to form a methoxy group. Peaks 11 and 14 were the aglycone forms of peaks 6 and 9, respectively. These peaks were presumed to be $5,7,3^{\prime}, 4^{\prime}$-tetrahydroxy-6-methoxyflavanone and 5,7,4'-trihydroxy-6-methoxyflavanone. The detected flavanones were found as aglycones and glycosides, and showed various hydroxyl and methoxyl patterns (Supplementary Table 1). These two flavanone glycosides (peaks 1 and 3) were tentatively identified for the first time as new flavonoids from $S$. plebeia. We further proposed a common name for these flavanones as 5,7,4'-trihydroxy-6-methoxyflavanone 7-O-glucoside (naasalvinin A), 5,6,7,3',4'-pentahydroxyflavanone 7 - $O$-glucoside (naasalvinin $\mathrm{B}$ ), 


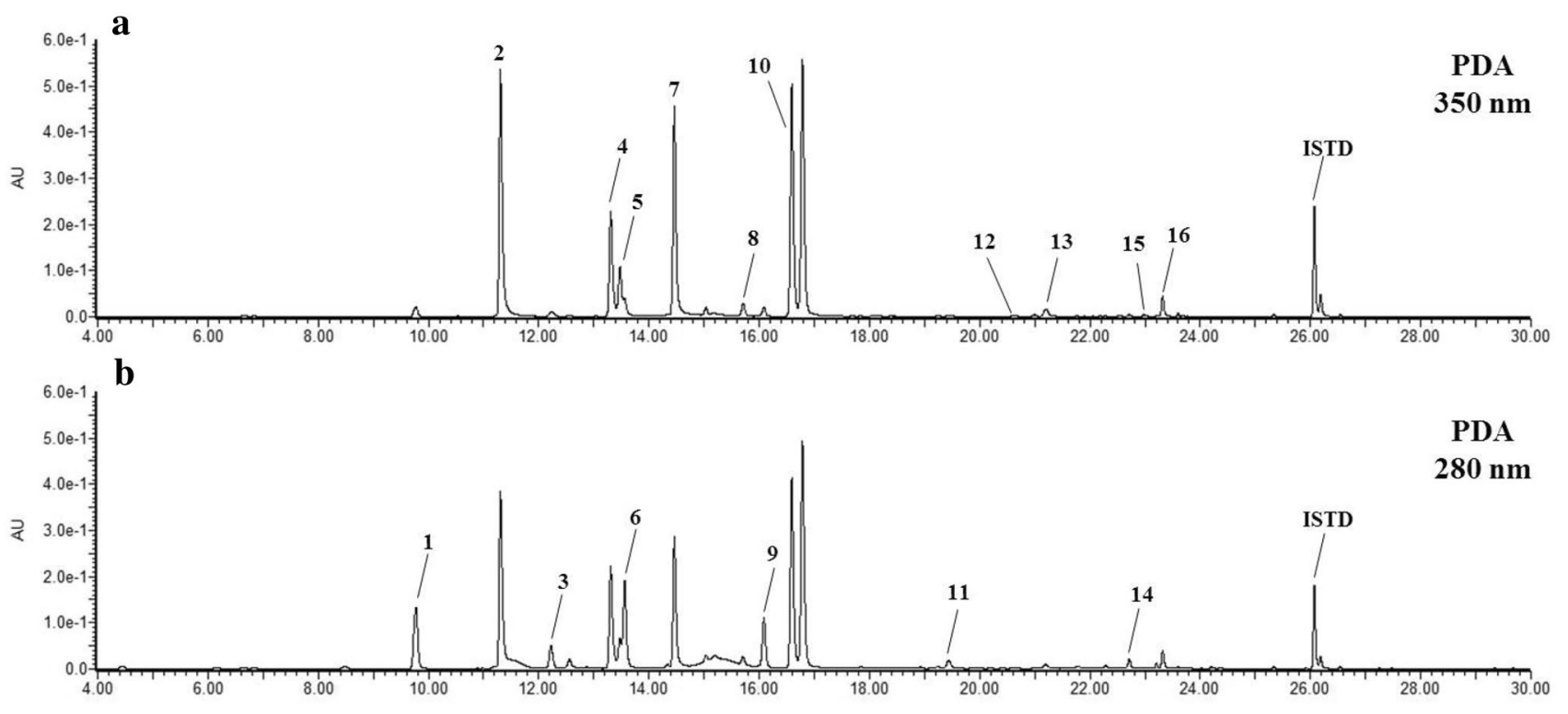

Fig. 1 UPLC-DAD chromatograms of phenolic compounds in S. plebeia $\mathrm{R}$. Br. a at $350 \mathrm{~nm}$ for flavones; and $\mathbf{b}$ at $280 \mathrm{~nm}$ for flavanones. (1) 5,6,7,3',4'-pentahydroxyflavanone 7-O-glucoside (naasalvinin B), (2) 6-hydroxyluteolin 7-O-glucoside, (3) 5,6,7,4'-tetrahydroxyflavanone 7- $O$-glucoside (naasalvinin $\mathrm{C}$ ), (4) luteolin 5-O-glucoside (galuteolin), (5) luteolin 7-O-glucoside (cynaroside), (6) 5,7,3',4'-tetrahydroxy-6-methoxyflavanone 7-O-glucoside (naasanone), (7) nepe- tin 7-O-glucoside (nepitrin), (8) apigenin 7-O-glucoside (cosmosiin), (9) 5,7,4'-trihydroxy-6-methoxyflavanone 7-O-glucoside (naasalvinin $\mathrm{A}),(10)$ hispidulin $7-O$-glucoside (homoplantaginin), (11) $5,7,3^{\prime}, 4^{\prime}$-tetrahydroxy-6-methoxyflavanone, (12) luteolin, (13) nepetin (6-methoxyluteolin), (14) 5,7,4'-trihydroxy-6-methoxyflavanone, (15) apigenin, (16) hispidulin (6-methoxyapigenin)
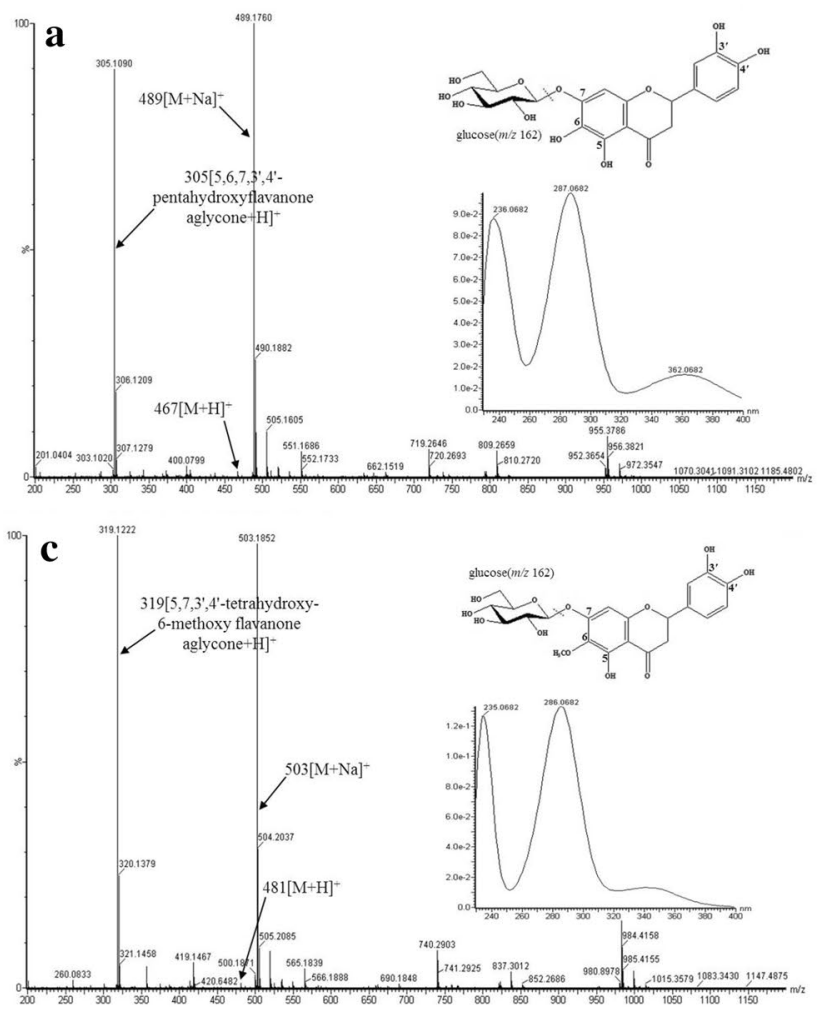

Fig. $2 \mathrm{MS} / \mathrm{UV}$ spectra and structures of four representative flavanones in S. plebeia $\mathrm{R}$. Br. a 5,6,7,3',4'-pentahydroxyflavanone 7-O-glucoside (naasalvinin B), b 5,6,7,4'-tetrahydroxyflavanone
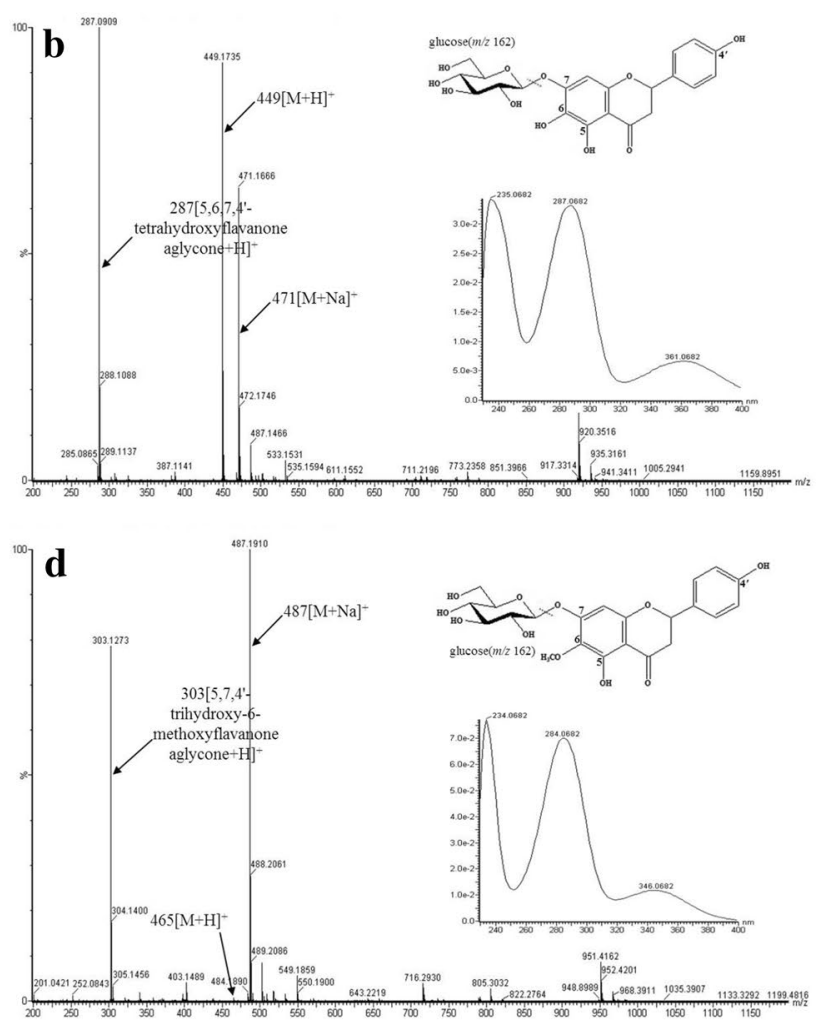

7-O-glucoside (naasalvinin C), c 5,7,3',4'-tetrahydroxy-6-methoxyflavanone 7-O-glucoside (naasanone), d 5,7,4'-trihydroxy-6-methoxyflavanone $7-O$-glucoside (naasalvinin $\mathrm{A}$ ) 

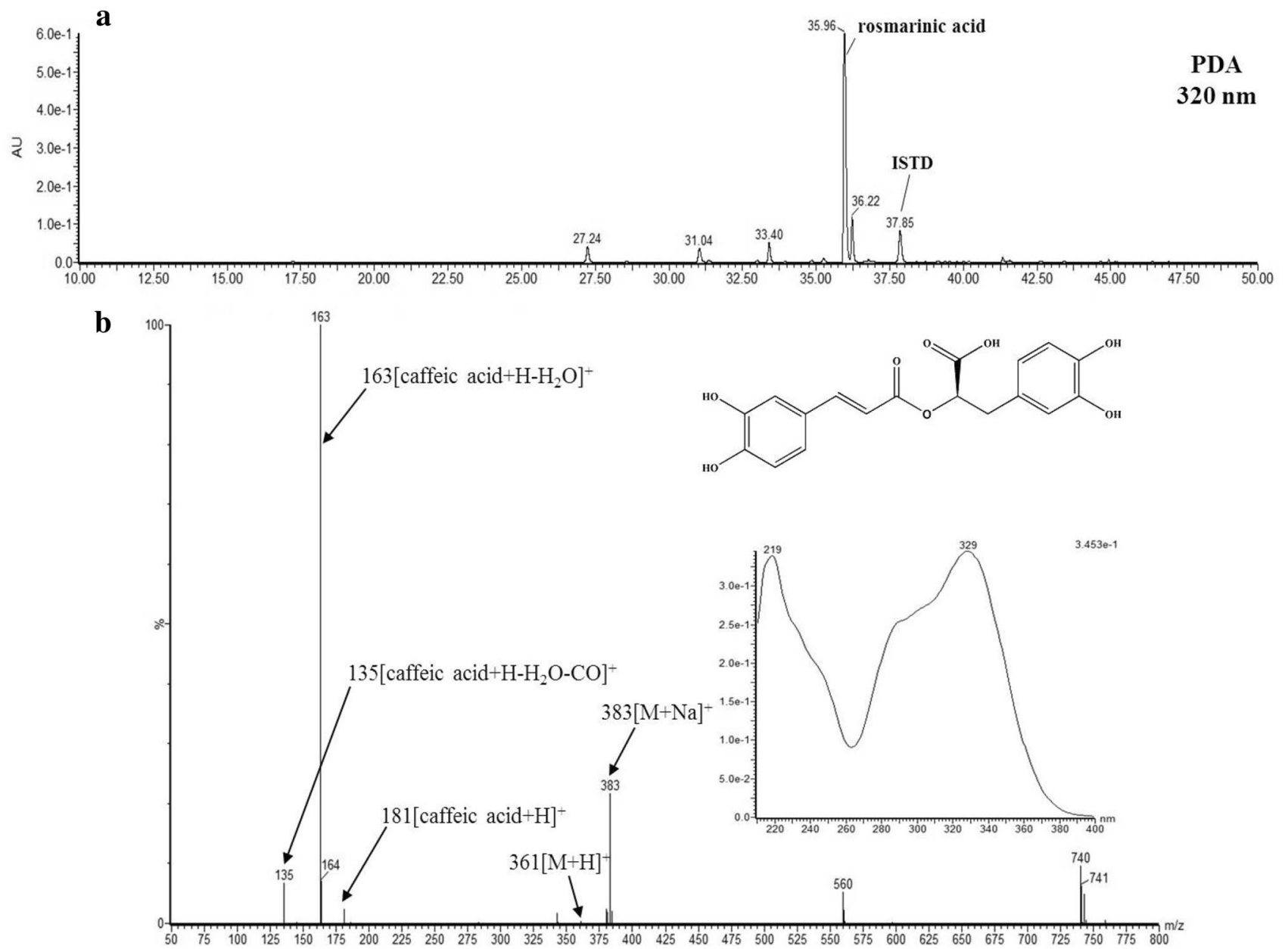

Fig. 3 UPLC-DAD chromatogram and MS/UV spectra of rosmarinic acid in S. plebeia $\mathrm{R}$. Br. a at $320 \mathrm{~nm}$ for RA; and $\mathbf{b}$ MS/UV spectra

5,6,7,4'-tetrahydroxyflavanone 7-O-glucoside (naasalvinin C) and 5,7,3',4'-tetrahydroxy-6-methoxyflavanone 7-O-glucoside (naasanone). Finally, flavonoids containing 6-hydroxyluteolin 7-O-glucoside and a series of naasalvinin were further required to separate single compounds and identify chemical structures using the NMR technique. However, further NMR data are required to clearly confirm the structures of the compounds.

In Salvia species, several flavanones have been reported, including 6-methoxynaringenin, 6-methoxynaringenin 7-O-glucoside, and filifolin, which have methoxy patterns at the 6-position of the flavanone backbone, except for eriodictyol $[3,26]$. However, in our study, we did not detect these compounds, perhaps because of agro-ecological variations in plant growth, maturation stage, and/or the analytical methods used to extract the flavonoids [26-28].

As shown in Table 1, 6-hydroxyluteolin 7-O-glucoside, hispidulin 7-O-glucoside (homoplantaginin), and nepetin 7- $O$ glucoside (nepetrin) were the major flavone compounds quantified, having concentrations of 2,452.7, 2,281.0, and
2,002.6 mg/100 g dry weight (DW), respectively, which accounted for $58.4 \%$ of the total flavonoids in the plant. The higher concentration of homoplantaginin, which indicates that this flavonoid is the major flavone in S. plebeia is inconsistence with previous reports [9]. 6-Hydroxyluteolin 7-O-glucoside was previously reported in plants of the Lamiaceae family as a major flavone glycoside $[29,30]$. Luteolin 5-O-glucoside (galuteolin), naasanone, and naasalvinin B were also identified in significant amounts and quantified as 1,101.1, 938.3, and $775.1 \mathrm{mg} / 100 \mathrm{~g} \mathrm{DW}$, respectively.

The amount of flavonoids existing in the plant in aglycone forms was small, accounting for only $4.3 \%$ of the total flavonoids. In the genus Salvia, reasonable amounts of different types of flavonoids were identified. For example, apigenin $(250 \mathrm{mg} / 100 \mathrm{~g}$ dry weight extracts; DWE) and its glycoside (360 mg/100 g DWE), isorhamnetin (290 mg/100 g DWE), and hispidulin $(630 \mathrm{mg} / 100 \mathrm{~g}$ DWE) were reported from the ethyl acetate extract of S. officinalis [31]. Similarly, nepitrin, homoplantaginin, nepetin, and hispidulin were also reported $1,215,1,050,792$, and $788 \mathrm{mg} / 100 \mathrm{~g}$ DWE, respectively, 


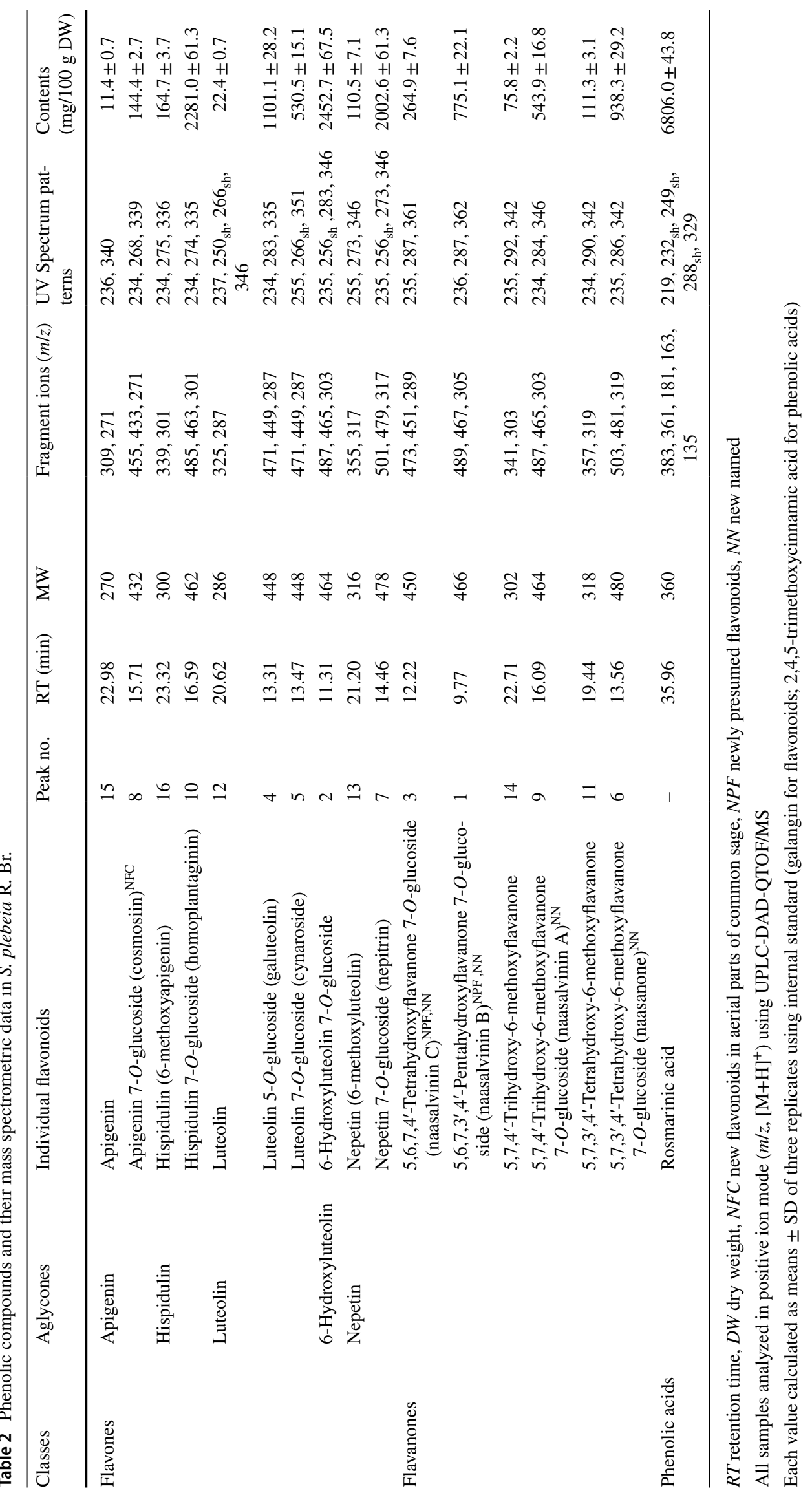


from the methanol extract of the plant [9]. Moreover, higher concentrations of cynaroside, nepitrin, homoplantaginin, nepetin, and hispidulin were identified from the ethanol extract of S. plebeia [16].

The abundance of luteolin 7-O-glucoside was found to be twofold higher than that of luteolin 5-O-glucoside. The natural glycosylation process in the plant was most likely to occur at $7^{\prime}-, 3^{\prime}-$, and $4^{\prime}-O$-glucoside positions than the 5 '-O-glucoside position. However, the present study indicated that the A-ring of the flavonoid is potentially conjugated at the 7-hydroxyl group of the sugar molecule [32]. The $O$-methoxylated flavonoids are also substituted at the 6-position of aglycones, such as nepetin. Based on the percentages of flavones $(76.5 \%)$ and flavanones $(23.5 \%)$ from total flavonoids, the former was predominant. Flavanones are natural primary biosynthetic intermediates for the synthesis of flavones [33]. Thus, the abundance of flavones may represent the utilization of flavanones as precursors for the biosynthesis of the flavones identified in S. plebeia.

Pharmacological activities of flavones have been reported in cell and animal model-based evaluations of individual compounds from S. plebeia. For example, luteolin and hispidulin had inhibitory effects on cytokine production in a postmenopausal osteoporosis model [28]. The 6-methoxyflavones, such as hispidulin and nepetin, were also reported as having antiviral activities against influenza A neuraminidase [27]. In particular, hispidulin, a marker compound in S. plebeia exhibited strong antioxidant, anti-fungal, antimutagenic, and anti-neoplastic properties [34, 35]. Even though, there was no report on the medicinal effect related to flavanone from S. plebeia; antioxidant [36], anti-tumoral [17], anti-diabetic [37], and anti-obesity [38] activities have been reported on this compound identified from Citrus spp. Flavanones could be a potential nutraceutical candidates [17, 39]. Thus, flavanones, such as naasalvinin $B$ and $C$ from Korean common sage, create unique flavanone derivatives that might be candidate compounds to treat chronic diseases.

\section{Identification and quantification of phenolic acids from S. plebeia R. Br.}

The hydrocinnamic derivative of phenolic acids besides the flavonoids was also quantitatively determined from $S$. plebeia. This peak was a predominant peak in the chromatogram compared to the other flavonoid peaks (Fig. 1a, b). The mass fragmentation revealed pseudomolecular ion $[\mathrm{M}+\mathrm{Na}]^{+}$ at $m / z 383,[\mathrm{M}+\mathrm{H}]^{+}$at $m / z 361$, and three product ions at $\mathrm{m} / \mathrm{z}$ 181(-180 mu, caffeic acid), $\mathrm{m} / \mathrm{z}, 163(-198 \mathrm{mu}$, further loss of a $\mathrm{H}_{2} \mathrm{O}$ ), and $\mathrm{m} / \mathrm{z} 135(-226 \mathrm{mu}$, further loss of a $\mathrm{CO}$ ). Hence comparing its MS data with the literature this peak is identified as rosmarinic acid (RA) (Fig. 3) [3, 23, 26, 33]. RA and its derivatives were reported to be widely distributed in Lamiaceae family [40]. The previous studied were also stated that the presence of phenolic acids and its derivatives such as, protocatechuic acid, caffeic acid, syringic acid, vanillic acid, ethyl caffeate, and methyl rosmarinate $[3,11$, 19, 26-28, 30]. In similar with previous studies higher concentration of RA was determined $(6806.0 \mathrm{mg} / 100 \mathrm{~g} \mathrm{DW})$. The higher concentration of RA in this plant could make S. plebeia as a potential functional ingredient in cosmetic industry [40].

\section{Conclusions}

A total of 16 flavonoid compounds and 1 phenolic acid were quantitatively identified from S. plebeia. Flavonoids were classified into 10 flavones and 6 flavanones. Apigenin, hispidulin, and luteolin were identified from this plant. The hydroxy- and methoxy-derivatives of luteolin were also determined. 6-Hydroxyluteolin 7-O-glucoside was predominant flavonoids and assumed to be a chemo-marker for this plant. Four new compounds among the flavanones were identified from S. plebeia for the first time and named as naasanone and naasalvinin A, B, and C. RA was also identified as representative phenolic acid. Thus, from the data the presence of higher concentration of polyphenolic constitutes in S. plebeia supported the potential medicinal capabilities of the plant.

Acknowledgements This study was carried out with the support of "Research (Project No. 012658)", National Institute of Agricultural Science, Rural development Administration, Republic of Korea.

Open Access This article is distributed under the terms of the Creative Commons Attribution 4.0 International License (http://creativeco mmons.org/licenses/by/4.0/), which permits unrestricted use, distribution, and reproduction in any medium, provided you give appropriate credit to the original author(s) and the source, provide a link to the Creative Commons license, and indicate if changes were made.

\section{References}

1. Embuscado ME (2015) Spices and herbs: natural sources of antioxidants: a mini review. J Funct Foods 18:811-819

2. Lu Y, Foo LY (2002) Polyphenolics of Salvia-a review. Phytochem 59:117-140

3. Lee SJ, Jang HJ, Kim Y, Oh HM, Lee S, Jung K, Rho MC (2016) Inhibitory effects of IL-6-induced STAT3 activation of bio-active compounds derived from Salvia plebeia $\mathrm{R}$. Br. Process Biochem 51:2222-2229

4. Peng MM, Fang Y, Hu W, Huang Q (2010) The pharmacological activities of compound Salvia plebeia granules on treating urinary tract infection. J Ethnopharmacol 129:59-63

5. Gu L, Weng X (2001) Antioxidant activity and components of Salvia plebeia R. Br.-a Chinese herb. Food Chem 73:299-305

6. Weng XC, Wang W (2000) Antioxidant activity of compounds isolated from Salvia plebeia. Food Chem 71:489-493

7. Nugroho A, Kim MH, Choi J, Baek NI, Park HJ (2012) In vivo sedative and gastroprotective activities of Salvia plebeia 
extract and its composition of polyphenols. Arch Pharm Res 35:1403-1411

8. Shin TY, Lee KB, Kim SH (2002) Anti-allergic effects of Sanguisorba officinalis on animal models of allergic reactions. Immunopharmacol Immunotoxicol 24:455-468

9. Akram M, Syed AS, Kim KA, Lee JS, Chang SY, Kim CY, Bae O (2015) Heme oxygenase 1-mediated novel anti-inflammatory activities of Salvia plebeia and its active components. J Ethnopharmacol 174:322-330

10. Choi JK, Oh HM, Lee S, Kwon TK, Shin TY, Rho MC, Kim SH (2014) Salvia plebeia suppresses atopic dermatitis-like skin lesions. Am J Chin Med 42:967-985

11. Jin XF, Lu YH, Wei DZ, Wang ZT (2008) Chemical fingerprint and quantitative analysis of Salvia plebeia $\mathrm{R}$. Br. by high-performance liquid chromatography. J Pharm Biomed Anal 48:100-104

12. Garcia-Lafuente A, Guillamon E, Villares A, Rostagno MA, Martinez JA (2009) Flavonoids as anti-inflammatory agents: implications in cancer and cardiovascular disease. Inflamm Res 58:537-552

13. Kawser Hossain M, Abdal Dayem A, Han J, Yin Y, Kim K, Kumar Saha S, Cho SG (2016) Molecular mechanisms of the anti-obesity and anti-diabetic properties of flavonoids. Int J Mol Sci 17:569

14. Jin MR, Xu H, Duan CH, Chou GX (2015) Two new flavones from Salvia plebeia. Nat Prod Res 29:1315-1322

15. Qu XJ, Xia X, Wang YS, Song MJ, Liu LL, Xie YY, Gao JJ (2009) Protective effects of Salvia plebeia compound homoplantaginin on hepatocyte injury. Food Chem Toxicol 47:1710-1715

16. Jin XF, Qian J, Lu YH (2011) The role of hepatoprotective effect of a flavonoid-rich extract of Salvia plebeia $\mathrm{R}$. Br. on carbon tetrachloride-induced acute hepatic injury in mice. J Med Plant Res 5:1558-1563

17. Cabrera M, Simoens M, Falchi G, Lavaggi ML, Piro OE, Castellano EE, Sagrera G (2007) Synthetic chalcones, flavanones, and flavones as antitumoral agents: biological evaluation and structure: activity relationships. Bioorg Med Chem 15:3356-3367

18. Kim HW, Kim JB, Cho SM, Chung MN, Lee YM, Chu SM, Kim JH (2012) Anthocyanin changes in the Korean purple-fleshed sweet potato, Shinzami, as affected by steaming and baking. Food chem 130:966-972

19. Ren DB, Qin YH, Yun YH, Lu HM, Chen XQ, Liang YZ (2014) Separation of nine compounds from Salvia plebeia $\mathrm{R}$. Br. using two-step high-speed counter-current chromatography with different elution modes. J Sep Sci 37:2118-2125

20. Jiang AL, Wang CH (2006) Antioxidant properties of natural components from Salvia plebeia on oxidative stability of ascidian oil. Process Biochem 41:1111-1116

21. Del Rio D, Stewart AJ, Mullen W, Burns J, Lean ME, Brighenti F, Crozier A (2004) HPLC-MS ${ }^{n}$ analysis of phenolic compounds and purine alkaloids in green and black tea. J Agric Food Chem 52:2807-2815

22. Jin Q, Han X, Hwang J, Hong S, Park M, Lee C, Hwang B (2009) A new phenylbutanone glucoside from Salvia plebeia. Nat Prod Sci 15:106-109

23. Grayer RJ, Kite GC, Abou-Zaid M, Archer LJ (2000) The application of atmospheric pressure chemical ionisation liquid chromatography-mass spectrometry in the chemotaxonomic study of flavonoids: characterisation of flavonoids from Ocimum gratissimum var. gratissimum. Phytochem Anal 11:257-267
24. Pereira OR, Silva AM, Domingues MR, Cardoso SM (2012) Identification of phenolic constituents of Cytisus multiflorus. Food Chem 131:652-659

25. Hostetler GL, Riedl KM, Schwartz SJ (2011) Endogenous enzymes, heat, and $\mathrm{pH}$ affect flavone profiles in parsley (Petroselinum crispum var. neapolitanum) and celery (Apium graveolens) during juice processing. J Agric Food Chem 60:202-208

26. Lee CT, Lee JN, Hong JT (2010) Phytochemical constituents from Salvia plebeia. Nat Prod Sci 16:207-210

27. Bang S, Ha TKQ, Lee C, Li W, Oh WK, Shim SH (2016) Antiviral activities of compounds from aerial parts of Salvia plebeia $\mathrm{R}$. Br. J Ethnopharmacol 192:398-405

28. Kim MH, Jung K, Nam KH, Jang HJ, Lee SW, Kim Y, Rho MC (2016) Salvia plebeia R. Br. inhibits signal transduction of IL-6 and prevents ovariectomy-induced bone loss by suppressing osteoclastogenesis. Arch Pharm Res 39:1671-1681

29. Borrás-Linares I, Stojanović Z, Quirantes-Piné R, Arráez-Román D, Švarc-Gajić J, Fernández-Gutiérrez A, Segura-Carretero A (2014) Rosmarinus officinalis leaves as a natural source of bioactive compounds. Int J Mol Med Sci 15:20585-20606

30. Dent M, Dragovic-Uzelac V, Penic M, Brncic M, Bosiljkov T, Levaj B (2013) The effect of extraction solvents, temperature and time on the composition and mass fraction of polyphenols in Dalmatian wild sage (Salvia officinalis L.) extracts. Food Technol Biotechnol 51:84

31. Kontogianni VG, Tomic G, Nikolic I, Nerantzaki AA, Sayyad N, Stosic-Grujicic S, Tzakos AG (2013) Phytochemical profile of Rosmarinus officinalis and Salvia officinalis extracts and correlation to their antioxidant and anti-proliferative activity. Food Chem 136:120-129

32. Iwashina $\mathrm{T}(2000)$ The structure and distribution of the flavonoids in plants. J Plant Res 113:287-299

33. Martens S, Mithöfer A (2005) Flavones and flavone synthases. Phytochem 66:2399-2407

34. Patel K, Patel DK (2017) Medicinal importance, pharmacological activities, and analytical aspects of hispidulin: a concise report. J Tradit Complement Med 7:360-366

35. Jeon YJ, Kwak HY, Choi JG, Lee JH, Choi SI (2016) Analytical method for the validation of hispidulin as a marker compound for the standardization of Salvia plebeia $\mathrm{R}$. Br. extracts as a functional ingredient. Korean J Med Crop Sci 24:271-276

36. Di Majo D, Giammanco M, La Guardia M, Tripoli E, Giammanco S, Finotti E (2005) Flavanones in Citrus fruit: structure-antioxidant activity relationships. Food Res Int 38:1161-1166

37. Constantin RP, Constantin RP, Bracht A, Yamamoto NS, IshiiIwamoto EL, Constantin J (2014) Molecular mechanisms of citrus flavanones on hepatic gluconeogenesis. Fitoterapia 92:148-162

38. Yoshida H, Watanabe W, Oomagari H, Tsuruta E, Shida M, Kurokawa M (2013) Citrus flavonoid naringenin inhibits TLR2 expression in adipocytes. J Nutr Biochem 24:1276-1284

39. Zhang X, Khalidi O, Kim SY, Wang R, Schultz V, Cress BF, Linhardt RJ (2016) Synthesis and biological evaluation of 5,7-dihydroxyflavanone derivatives as antimicrobial agents. Bioorg Med Chem Lett 26:3089-3092

40. Petersen M, Simmonds MS (2003) Rosmarinic acid. Phytochem 62:121-125 


\section{Affiliations}

\section{Seon-Hye Lee ${ }^{1} \cdot$ Heon-Woong Kim ${ }^{1} \cdot$ Min-Ki Lee ${ }^{1} \cdot$ Young Jin Kim ${ }^{1} \cdot$ Gelila Asamenew $^{1} \cdot$ Youn-Soo Cha ${ }^{2}$. Jung-Bong $\mathrm{Kim}^{1}{ }^{\mathbb{C}}$}

1 Department of Agro-food Resources, National Institute of Agricultural Sciences, Rural Development Administration, 166, Nongsaengmyeong-ro, Iseo-myeon, Wanju-gun, Jeollabuk-do, Republic of Korea
2 Department of Food Science and Human Nutrition, Chonbuk National University, 567, Baekje-daero, Deokjin-gu,

Jeonju-si, Jeollabuk-do, Republic of Korea 